\title{
Projet parental des internes de médecine générale d'Aix-Marseille université : connaissances théoriques en reproduction et attitude vis- à-vis de la parentalité
}

\author{
Parental project of general medicine residents from Aix-Marseille university: \\ Theoretical knowledge in reproduction and behavior towards parenting
}

\author{
A. Fabregue ${ }^{a}$, B. Moheng ${ }^{b}$, A. Laynet $^{a}$, A. Agostini $^{\mathrm{b}, \mathrm{c}}$, L. Boubli $^{\mathrm{b}, \mathrm{c}}$, B. Courbiere $^{\mathrm{c}, *, \mathrm{~d}}$ \\ a Département universitaire de médecine générale, faculté de médecine, Aix-Marseille université, Campus Timone, 27, boulevard Jean-Moulin, \\ 13385 Marseille cedex 05, France \\ ${ }^{\mathrm{b}}$ Unité de recherche EA 3279, Santé publique et maladies chroniques, laboratoire de Santé Publique, faculté de médecine, université de la Méditerranée, 27, \\ boulevard Jean-Moulin, 13385 Marseille cedex 05, France \\ ${ }^{\mathrm{c}}$ Pôle Femmes-Parents-Enfants, hôpital Nord, hôpital de La Conception, AP-HM, 147, boulevard Baille, 13005 Marseille, France \\ ${ }^{\mathrm{d}}$ Aix-Marseille université, CNRS, IRD, Avignon université, IMBE UMR 7263, 13397 Marseille, France
}

\section{Mots clés :}

Fertilité

Connaissances

Parentalité

Internes en médecine

\section{R É S U M É}

Objectifs. - Les internes en médecine sont concernés par la problématique du report de leur projet parental du fait de la longueur de leurs études et de l'instabilité de leurs conditions de vie (stages semestriels de localisation et d'horaires variables, valorisation de la mobilité). Les médecins généralistes sont en première ligne pour informer les femmes en âge de procréer sur le déclin de la fertilité liée à l'âge. Nous avons souhaité étudier si les internes des deux sexes étaient assez sensibilisés au problème de la baisse physiologique de la fertilité féminine avec l'âge.

Méthodes. - Étude transversale menée de juin à septembre 2015 par auto-questionnaires envoyés à tous les internes de médecine générale d'Aix-Marseille université. Le questionnaire était composé de trois parties : (i) une partie descriptive permettant d'obtenir des informations générales et personnelles sur les internes et leurs intentions par rapport à la parentalité ; (ii) neuf questions testant leurs connaissances sur la fertilité et la réserve ovarienne ; (iii) une question simulée testant leur attitude supposée en cas de diagnostic de diminution de la réserve ovarienne chez l'interne féminine ou chez la compagne d'un interne masculin.

Résultats. - Sur les 631 internes de médecine générale, le taux de participation global a été de 53,2\%, avec 220 femmes âgées en moyenne de $27 \pm 1,8$ ans et de 116 hommes âgés en moyenne de 27,3 $\pm 2,2$ ans : 11,8\% des internes féminines $(n=26)$ et $6,9 \%$ des internes masculins $(n=8)$ avaient déjà un ou des enfants. Parmi les internes sans enfants, $99 \%$ des femmes $(n=192)$ et $91,7 \%$ des hommes $(n=99)$ affirmaient en vouloir dans le futur, en prévoyant un âge moyen pour être parents de 29,6 $\pm 1,9$ ans pour les femmes et 30,4 $\pm 2,2$ ans pour les hommes. Bien que les connaissances sur le déclin de la fertilité de la femme avec l'âge fussent majoritairement acquises, les internes déclaraient vouloir reporter leur projet parental après la fin des études médicales pour $58,3 \%$ des femmes $(n=112)$ et $53,5 \%$ des hommes $(n=53)$. Les capacités de la fécondation in vitro à compenser les effets de lâge sur la fertilité de la femme étaient surestimées par 9 internes sur 10. Devant une mise en situation du couple face à une hypothèse de diminution de la réserve ovarienne, 73,6\% des femmes $(n=162)$ et $61,2 \%$ des hommes $(n=71)$ seraient prêts à modifier leurs choix de vie et à concevoir un enfant plus tôt que prévu. Les internes masculins étaient plus favorables que les femmes à l'autoconservation ovocytaire pour convenance personnelle afin de différer le projet de maternité ( $61,2 \%$ vs $46,8 \%$ ).

Conclusion. - Les internes en médecine générale ont pratiquement tous le projet d'avoir des enfants et plus de la moitié reportent leur projet parental après la fin de leur internat, bien que les connaissances sur le déclin de la fertilité de la femme avec l'âge sont globalement acquises.

\footnotetext{
* Auteur correspondant.

Adresse e-mail : blandine.courbiere@univ-amu.fr (B. Courbiere).
} 
Keywords

Fertility

Knowledge

Parenting

Residency
Objectives. - Analyze residents' intentions about parenting and knowledge about fertility, as well as their alleged behavior towards a diagnosis of diminished ovarian reserve.

Materials and methods. - Prospective study with distribution of self-administered questionnaire to residents of Aix-Marseille university from June to September 2015. The questionnaire was composed of a first descriptive part including generic and personal informations about the residents and their intentions towards parenting. The second part was a 9 questions survey aimed at testing their knowledge about fertility and ovarian reserve and a final question placing them in a situation of a diminished ovarian reserve diagnosis in order to analyze their reactions.

Results. - The overall participation rate was 53.2\%, including 220 women with an average age of 27 years (SD: 1.8 years) and 116 men with an average age of 27.3 years (SD: 2.2 years). The $11.8 \%$ of female residents $(n=26)$ already had one or more children $v s .6 .9 \%$ of male residents $(n=8)$. Among the residents without children, $99 \%$ of females $(n=192)$ and $91.7 \%$ of males $(n=99)$ said they wanted to have children in the future, planning an average age to have their first child of 29.6 years (SD: 1.9 years) for females and 30.4 years (SD: 2.2 years) for men. Moreover, $58.3 \%$ of female residents $(n=112)$ and $53.5 \%$ of male residents $(n=53)$ reported that they were postponing their plan to have children after the completion of their medical studies. In a simulation of couples facing a diagnosis of diminished ovarian reserve, $73.6 \%$ of females and $61.2 \%$ of males would be ready to change their life choices and conceive a baby sooner than originally planned.

Conclusion. - Almost all the general medicine residents have plannedto have children in the future and more than half of them postpone their parental plan after the end of their residency, although the decline of female fertility in relation to aging is widely known among them.

\section{Introduction}

La fertilité d'une femme diminue dès l'âge de 31 ans du fait d'une baisse physiologique de sa réserve ovarienne et cette diminution s'accélérerait après 37 ans [1,2]. En France, en 2014, l'âge moyen des mères à leur accouchement était de 30,3 ans tous rangs de naissances confondus alors qu'il était de 26 ans dans les années 1970. En parallèle, un couple sur cinq consulte en France pour infertilité et l'âge maternel apparaît comme être un des facteurs pronostiques principaux [3]. L'âge maternel est aussi paradoxalement souvent un facteur causal de l'infertilité d'un couple [4]. De nombreux couples pensent à tort que les techniques d'assistance médicale à la procréation (AMP) peuvent compenser le problème de baisse de la fertilité liée à l'âge. En cas de report de première grossesse de 30 à 35 ans, les techniques d'AMP ne rattraperaient que la moitié des grossesses perdues [5].

Le report de l'âge du premier enfant peut s'expliquer par de nombreuses raisons : la maîtrise de la contraception, l'allongement de la durée des études et la gestion des carrières professionnelles chez des femmes craignant le redouté " plafond de verre ", la recherche d'un travail stable, une situation financière difficile... Les internes en médecine constituent une population concernée par la problématique du report de leur projet parental de par la longueur de leurs études (entre 9 et 13 ans selon la spécialité choisie) et de par l'instabilité de leurs conditions de vie (stages semestriels de localisation et d'horaires variables, valorisation de la mobilité). Ainsi, les femmes diplômées du supérieur ont leur premier enfant, en moyenne 1,7 an plus tard que la moyenne des femmes résidant en France métropolitaine. Ce retard de la primiparité est encore plus marqué dans les régions île-de-France, Midi-Pyrénées et Provence-Alpes-Côte d'Azur où se concentrent une majorité de femmes diplômées [6].

Concernant les comportements et connaissances des étudiants de l'enseignement supérieur, il a été observé en population étudiante "tous horizons " que les étudiantes envisageaient de reporter leur projet parental du fait de leurs études, qu'elles sous-estimaient l'âge de déclin de la fertilité féminine et surestimaient les chances de succès des techniques d'AMP. Sur une population d'étudiants en médecine, Nouri et al. (2014) [7] ont montré qu'ils prévoyaient d'avoir des enfants plus tard que les étudiants en parcours non médicaux et bien qu'en ayant plus conscience de l'effet de l'âge sur la fertilité, ces étudiants croyaient fortement que cet effet pouvait être compensé par les techniques d'AMP.

Les attitudes et savoirs des futurs médecins généralistes sont très intéressants à évaluer, car ce sont eux qui seront en première ligne avec les patientes, et qui auront un rôle majeur de prévention et d'information. Beaucoup de médecins généralistes assurent le suivi gynécologique de routine. En cas de fausse croyance (exemple : la FIV pallie à l'infertilité liée à l'âge) ou s'ils ne sont pas persuadés du bienfondé d'une information, le risque que l'information ne soit pas transmise aux femmes est élevé. Les spécialistes en médecine de la reproduction ne sont souvent consultés que lorsque la femme est déjà infertile, avec l'infertilité liée à l'âge comme motif de consultation fréquent, parfois par perte de temps du fait d'un recours trop tardif à un spécialiste de la part du médecin traitant... Nous nous sommes ainsi intéressés à l'attitude des internes de médecine générale concernant leur projet parental pour évaluer leur niveau de sensibilisation au problème de la baisse physiologique de leur fertilité avec l'âge pour les femmes ou de la fertilité de leur compagne. D'autre part, la plupart des études sur la perception de la fertilité ne s'intéressent qu'aux étudiantes de sexe féminin. Nous avons choisi d'étudier et de comparer les femmes et les hommes, en posant l'hypothèse que la perception des internes masculins pouvait être différente de celle de leurs consœurs. En pratique, l'homme est moins confronté à une " horloge biologique procréative " que la femme et peut parfois être à l'origine lui du report du projet parental du couple.

L'objectif de notre travail a été triple au sein de la population des internes en médecine générale d'Aix-Marseille université :

- évaluer leur intention en fonction de leur sexe concernant une éventuelle future parentalité ;

- tester par questionnaire leurs connaissances sur la fertilité et la réserve ovarienne ;

- étudier leur attitude en situation simulée de diagnostic de diminution de la réserve ovarienne chez l'interne féminine ou chez la compagne d'un interne masculin. 


\section{Matériels et méthodes}

Une étude transversale monocentrique a été menée de juin à septembre 2015 au sein de la faculté de médecine d'AixMarseille université avec envoi par mail à tous les internes de médecine générale d'un lien pour accéder à un auto-questionnaire anonyme en ligne. La liste des mails était exhaustive car fournie par le département universitaire de médecine générale. Les internes ont été informés en amont lors des choix semestriels de stage d'interne du futur déroulement de l'étude. Un auto-questionnaire par sexe a été réalisé en s'appuyant sur plusieurs questionnaires publiés dans des études sur cette thématique dans d'autres pays [7-9]. Cette étude a reçu un avis favorable du Comité d'éthique d'Aix-Marseille université (201602-03-004).

Les internes inclus étaient les internes de médecine générale tous sexes confondus qui avaient débuté leur internat entre 2012 et 2014 et qui étaient inscrits à Aix-Marseille université. Les internes des autres spécialités médicales et chirurgicales de la même université n'ont pas été inclus.

Le questionnaire (disponible en annexes) était composé de trois parties :

- une première partie descriptive avec des informations générales et personnelles non identifiantes des internes (âge, semestre d'internat, vie en couple ou non, durée de vie de couple, nombre d'enfants) et des questions concernant leurs intentions par rapport à leur projet de parentalité : combien d'enfant(s) souhaité(s), âge souhaité pour devenir parent, projet ou non de différer le projet parental après la fin des études médicales ; et si oui, recherche des raisons (raisons financières, difficultés professionnelles, volonté de clôturer les études, instabilité du couple, difficultés prévisibles de garde de l'enfant, peur d'être mal vue par l'entourage professionnel, demande d'un poste d'assistant-chef de clinique (CCA) et peur de ne pas l'obtenir, attente de l'installation en libéral, désir de garder une liberté de mobilité, autres centres d'intérêt (voyages...). Il a aussi été demandé si les internes se sentaient suffisamment informés des moyens mis à leur disposition (stage en surnombre, disponibilité, congés maternités-paternités) pour réaliser un projet de grossesse pendant leur internat de médecine?

- une seconde partie constituée de 9 questions afin de tester leurs connaissances sur la fertilité et la réserve ovarienne. Les questions théoriques posées étaient : à quel âge la fertilité naturelle décline chez une femme ? (vrai/faux, réponse attendue : 35 ans) ; fumer risque d'altérer la réserve ovarienne (oui/non) ; les contraceptifs oraux ont des effets délétères sur la réserve ovarienne (oui/non), la régularité des cycles menstruels est le marqueur d'une réserve ovarienne normale (oui/non), la fécondation in vitro permet à une femme de concevoir même avec une réserve ovarienne considérablement diminuée (oui/non), la réserve ovarienne peut varier considérablement chez les femmes (oui/non), il a été démontré que l'activité physique et une alimentation saine ont une action bénéfique sur la préservation de la réserve ovarienne (oui/ non), chaque femme dispose d'une réserve ovarienne fixe et non renouvelable dès la naissance (oui/non) ; quelle est la probabilité d'obtenir une grossesse au cours d'un cycle menstruel chez une femme de 35 ans ? (vrai/faux, réponse attendue : entre 10 et $20 \%$ );

- une troisième partie demandant aux femmes si elles seraient intéressées de connaître leur réserve ovarienne et pour les deux sexes, si leur attitude changeraient vis-à-vis de la parentalité en cas de diagnostic de diminution de la réserve ovarienne chez la femme ou chez leur compagne pour les hommes (mesurées à l'aide d'une échelle de Likert à 5 points). Il a aussi été demandé si les internes étaient favorables à la possibilité d'autoconserver ses ovocytes pour convenance personnelle afin de permettre aux femmes de différer leur projet de maternité.

Analyse statistique : les données recueillies étaient anonymes et ont été retranscrites sur un fichier à partir du logiciel Microsoft Excel@. L'analyse statistique a été réalisée avec le logiciel IBM SPSS statistics 20.0. Les variables quantitatives ont été présentées en moyenne \pm écart-type car la distribution des données quantitatives ne présentait pas d'écart à la normalité : une vérification graphique ainsi que le test de Kolmogorov Smirnov ont été réalisées. Les tests réalisés ont été le test $t$ de comparaison de moyenne (test paramétrique) pour comparer des variables quantitatives, le test du $\mathrm{Chi}^{2}$ de Pearson pour comparer deux variables qualitatives et le test exact de Fisher lorsqu'au moins une cellule avait un effectif théorique inférieur à 5 pour les petits effectifs. Pour tous les tests, la significativité était fixée à $p<0,05$.

\section{Résultats}

\section{Caractéristiques de la population étudiée}

Les questionnaires ont été distribués aux 631 internes de médecine générale (composés de 383 femmes et 248 hommes), le taux de participation total a été de 53,2 \% (336 internes participants tous sexes confondus) et a été de $57,4 \%$ chez les femmes ( $n=220$ réponses) et de $46,8 \%$ chez les hommes ( $n=116$ ). Le Tableau 1 présente les caractéristiques des internes inclus dans l'étude et leurs intentions concernant la parentalité. L'âge moyen des femmes était de $27 \pm 1,8$ ans et pour les hommes de $27,3 \pm 2,2$ ans. Parmi les internes féminines, 74,5\% d'entre elles étaient en couple et $11,8 \%$ d'entre elles $(n=26)$ avaient déjà un ou des enfants (conçus pendant l'internat pour la plupart). Concernant les hommes, 69 \% d'entre eux étaient en couple et 6,9 \% d'entre eux avaient déjà un ou des enfants (conçus pour la plupart pendant le $2^{\mathrm{e}}$ cycle ou l'internat). Parmi les internes sans enfants, $99 \%$ des femmes et 91,7 \% des hommes sans enfants affirmaient vouloir des enfants dans le futur, en prévoyant un âge moyen respectif pour être parents de $29,6 \pm 1,9$ ans et $30,4 \pm 2,2$ ans.

\section{Études de médecine et projet parental}

Sur les 336 internes interrogés, 58,3\% des femmes et 53,5\% des hommes affirmaient vouloir reporter leur projet parental après la fin des études médicales ( $3^{\mathrm{e}}$ cycle/internat validé) pour des raisons variées : des raisons financières pour $42,5 \%$ des femmes et $54,9 \%$ des hommes, des difficultés professionnelles pour 60,4\% des femmes et 62 \% des hommes, une volonté de clôturer les études pour $87,2 \%$ des femmes et $84,3 \%$ des hommes, une instabilité du couple pour $24,8 \%$ des femmes et $36,7 \%$ des hommes, des difficultés prévisibles de garde de l'enfant pour $67,9 \%$ des femmes et $68 \%$ des hommes, une peur d'être mal vue par l'entourage professionnel pour 7,9\% des femmes et $8,3 \%$ des hommes, une demande de poste de CCA et une peur de ne pas l'obtenir pour $10,9 \%$ des femmes et $18,8 \%$ des hommes, une attente de l'installation en libéral pour $37,9 \%$ des femmes et $45,1 \%$ des hommes, un désir de garder une liberté de mobilité pour 70,8\% des femmes et $66,7 \%$ des hommes et pour d'autres centres d'intérêts (voyages...) pour $77,8 \%$ des femmes et $59,6 \%$ des hommes.

Parmi les internes de médecine générale, 65,9\% des femmes et $75,9 \%$ des hommes ont estimé n'être pas assez informés des moyens mis à leur disposition (stage en surnombre, disponibilité, congés maternités et paternités) pour favoriser un projet d'enfant pendant leur internat de médecine. 
Tableau 1

Caractéristiques de la population étudiée et intentions concernant la parentalité.

Characteristics of general medicine residents and intentions of being parents.

\begin{tabular}{|c|c|c|c|}
\hline & Réponses & Femmes $(n=220)$ & Hommes $(n=116)$ \\
\hline Âge (moyenne en années) & & $27,0 \pm 1,8$ & $27,3 \pm 2,2$ \\
\hline \multirow[t]{4}{*}{ Semestre d'internat actuel (\%) } & $1^{\mathrm{er}}$ semestre & $0(0)$ & $0(0)$ \\
\hline & $2^{\mathrm{e}}$ semestre & $65(29,5)$ & $37(31,9)$ \\
\hline & $3^{\mathrm{e}}$ semestre & $12(5,5)$ & $17(14,7)$ \\
\hline & $4^{\mathrm{e}}$ semestre & $74(33,6)$ & $37(31,9)$ \\
\hline \multirow{2}{*}{$\begin{array}{l}\text { Pendant votre internat, avez-vous fait un stage ou suivi des consultations } \\
\text { de gynécologie-obstétrique? }(\%)\end{array}$} & Oui & $93(42,3)$ & $51(44)$ \\
\hline & Non & $127(57,7)$ & $65(56)$ \\
\hline \multirow[t]{2}{*}{ Êtes-vous en couple ? $(\%)$} & Oui & $164(74,5)$ & $80(69)$ \\
\hline & Non & $56(25,5)$ & $36(31)$ \\
\hline Si oui, depuis combien de temps ? (moyenne en mois) & & $48,0 \pm 37,5$ & $36,1 \pm 33,1$ \\
\hline \multirow[t]{2}{*}{ Avez-vous des enfants? (\%) } & Oui & $26(11,8)$ & $8(6,9)$ \\
\hline & Non & $194(88,2)$ & $108(93,1)$ \\
\hline \multirow[t]{3}{*}{ Si oui, à quelle période les avez-vous eu ? (\%) } & $1^{\mathrm{er}}$ cycle & $0(0)$ & $2(25)$ \\
\hline & $2^{\mathrm{e}}$ cycle & $3(11,5)$ & $3(37,5)$ \\
\hline & Internat & $23(88,5)$ & $3(37,5)$ \\
\hline \multirow[t]{5}{*}{ Nombre d'enfants souhaités pour les étudiants déjà parents (\%) } & 1 & $4(16)$ & $0(0)$ \\
\hline & 2 & $7(28)$ & $2(25)$ \\
\hline & 3 & $12(48)$ & $3(37,5)$ \\
\hline & 4 & $1(4)$ & $3(37,5)$ \\
\hline & 5 et plus & $1(4)$ & $0(0)$ \\
\hline \multirow[t]{2}{*}{ Pensez-vous que vous voudrez avoir des enfants dans le futur ? (\%) } & Oui & 192(99) & $99(91,7)$ \\
\hline & Non & $2(1)$ & $9(8,3)$ \\
\hline \multirow[t]{5}{*}{ Si oui, combien en souhaiteriez-vous ? (\%) } & 1 & $7(3,7)$ & $2(2,1)$ \\
\hline & 2 & $101(52,9)$ & $43(44,3)$ \\
\hline & 3 & $61(31,9)$ & $41(42,3)$ \\
\hline & 4 & $18(9,4)$ & $9(9,3)$ \\
\hline & 5 et plus & $4(2,1)$ & $2(2,1)$ \\
\hline Âge souhaité pour le $1^{\mathrm{er}}$ enfant (moyenne en années) & & $29,6 \pm 1,9$ & $30,4 \pm 2,2$ \\
\hline
\end{tabular}

\section{Connaissances sur la fertilité et la réserve ovarienne}

Le Tableau 2 présente les réponses données par les internes au questionnaire théorique sur la fertilité et la réserve ovarienne. Au total, 89,1\% des internes féminines et 91,4\% des hommes pensaient à tort que la fécondation in vitro permet à une femme de concevoir même avec une réserve ovarienne considérablement diminuée.
Comportement présumé devant un diagnostic de baisse de la réserve ovarienne

Le Tableau 3 expose les réponses des internes féminines à la mise en situation d'un diagnostic de baisse de la fertilité et le Tableau 4, les réponses des internes masculins à une mise en situation d'un diagnostic d'insuffisance ovarienne chez leur

Tableau 2

Réponse des internes au questionnaire sur la fertilité et la réserve ovarienne. Residents' answers about theorical test concerning fertility.

\begin{tabular}{|c|c|c|c|c|c|c|}
\hline & Réponses & $\begin{array}{l}\text { Nombre de } \\
\text { réponses } \\
\text { correctes } \\
\text { femmes (\%) }\end{array}$ & $\begin{array}{l}\text { Nombre de } \\
\text { réponses } \\
\text { correctes } \\
\text { hommes (\%) }\end{array}$ & $\begin{array}{l}\text { Nombre de } \\
\text { réponses } \\
\text { fausses } \\
\text { femmes (\%) }\end{array}$ & $\begin{array}{l}\text { Nombre de } \\
\text { réponses } \\
\text { fausses } \\
\text { hommes (\%) }\end{array}$ & $\begin{array}{l}\text { Valeur } \\
\text { de } p\end{array}$ \\
\hline À quel âge la fertilité naturelle décline chez une femme ? & 35 ans & $20(9,1)$ & $20(17,2)$ & $200(90,9)$ & $96(82,8)$ & 0,034 \\
\hline Fumer risque d'altérer la réserve ovarienne & Vrai & $205(93,2)$ & $107(92,2)$ & $15(6,8)$ & $9(7,8)$ & 0,824 \\
\hline $\begin{array}{l}\text { Les contraceptifs oraux ont des effets délétères sur la réserve } \\
\text { ovarienne }\end{array}$ & Faux & $155(70,5)$ & $78(67,2)$ & $65(29,5)$ & $38(32,8)$ & 0,619 \\
\hline $\begin{array}{l}\text { La régularité des cycles menstruels est le marqueur d'une réserve } \\
\text { ovarienne normale }\end{array}$ & Faux & $153(69,5)$ & $86(74,1)$ & $67(30,5)$ & $30(25,9)$ & 0,448 \\
\hline $\begin{array}{l}\text { La fécondation in vitro permet à une femme de concevoir même avec } \\
\text { une réserve ovarienne considérablement diminuée }\end{array}$ & Faux & $24(10,9)$ & $10(8,6)$ & $196(89,1)$ & $106(91,4)$ & 0,572 \\
\hline La réserve ovarienne peut varier considérablement chez les femmes & Vrai & $201(91,4)$ & $100(86,2)$ & $19(8,6)$ & $16(13,8)$ & 0,188 \\
\hline $\begin{array}{l}\text { Il a été démontré que l'activité physique et une alimentation saine ont } \\
\text { une action bénéfique sur la préservation de la réserve ovarienne }\end{array}$ & Faux & $107(48,6)$ & $40(34,5)$ & $113(51,4)$ & $76(65,5)$ & 0,015 \\
\hline $\begin{array}{l}\text { Chaque femme dispose d'une réserve ovarienne fixe et non } \\
\text { renouvelable dès la naissance }\end{array}$ & Vrai & $193(87,7)$ & $104(89,7)$ & $27(12,3)$ & $12(10,3)$ & 0,721 \\
\hline $\begin{array}{l}\text { Quelle est la probabilité d'obtenir une grossesse au cours d'un cycle } \\
\text { menstruel chez une femme de } 35 \text { ans? }\end{array}$ & $\begin{array}{l}\text { Entre } 10 \% \\
\text { et } 20 \%\end{array}$ & $68(30,9)$ & $41(35,3)$ & $152(69,1)$ & $75(64,7)$ & 0,462 \\
\hline
\end{tabular}

Tableau 3

Comportement présumé des femmes à la question " Dans l'hypothèse où une diminution de la réserve ovarienne vous était diagnostiquée, que feriez-vous ? ". Presumed behavior of women in case of a diagnosis of diminished ovarian reserve.

\begin{tabular}{|c|c|c|c|c|c|}
\hline & Tout à fait d'accord (\%) & D’accord (\%) & Ne sait pas (\%) & Pas d'accord (\%) & Pas du tout d'accord (\%) \\
\hline Se mettre en couple & $18(8,2)$ & $54(24,5)$ & $66(30)$ & $54(24,5)$ & $28(12,7)$ \\
\hline Avoir des enfants plus tôt que prévu & $79(35,9)$ & $83(37,7)$ & $36(16,4)$ & $17(7,7)$ & $5(2,3)$ \\
\hline Avoir recours à une autoconservation d'ovocytes & $31(14,1)$ & $70(31,8)$ & $74(33,6)$ & $30(13,6)$ & $15(6,8)$ \\
\hline Aucune incidence & $11(5)$ & $19(8,6)$ & $57(25,9)$ & $75(34,1)$ & $58(26,4)$ \\
\hline
\end{tabular}


Tableau 4

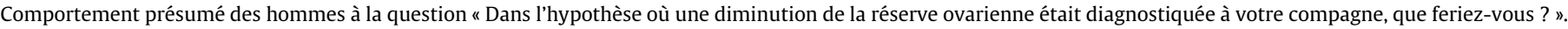
Presumed behavior of men if their partner was diagnosed with a diminished ovarian reserve.

\begin{tabular}{|c|c|c|c|c|c|}
\hline & Tout à fait d'accord (\%) & D'accord (\%) & Ne sait pas (\%) & Pas d'accord (\%) & Pas du tout d'accord (\%) \\
\hline Avoir des enfants plus tôt que prévu & $31(26,7)$ & $40(34,5)$ & $37(31,9)$ & $5(4,3)$ & $3(2,6)$ \\
\hline Lui conseiller une autoconservation d'ovocytes & $16(13,8)$ & $37(31,9)$ & $53(45,7)$ & $7(6)$ & $3(2,6)$ \\
\hline Aucune incidence & $5(4,3)$ & $19(16,4)$ & $56(48,3)$ & $22(19)$ & $14(12,1)$ \\
\hline
\end{tabular}

compagne. Parmi les internes, 61,2 \% des hommes et 73,6 \% des femmes seraient " tout à fait d'accord " ou " d'accord " pour modifier leurs choix de vie et concevoir un enfant plus tôt que prévu en cas de diagnostic de baisse de réserve ovarienne. Ce diagnostic n'aurait pas ou peu d'incidence sur l'initiation d'un projet parental pour $20,7 \%$ des hommes et $13,6 \%$ des femmes. Les internes masculins étaient plus favorables que les femmes à l'autoconservation ovocytaire pour convenance personnelle afin de différer le projet de maternité (61,2\% vs 46,8\%).

\section{Discussion}

Connaissances théoriques satisfaisantes malgré la persistance d'idées reçues

Nous avons observé que les internes de médecine générale sont globalement bien informés de la diminution de la fertilité féminine avec l'âge. Nouri et al. avait montré que les étudiants en médecine avaient une meilleure connaissance de l'impact de l'âge sur la fertilité féminine que les étudiants non médicaux [7]. Il aurait d'ailleurs été intéressant de réaliser la même étude avec un groupe contrôle en population générale pour apprécier les différences de connaissances. L'impact de l'intoxication tabagique sur la fertilité était connu de par plus de $90 \%$ des internes montrant les fruits de l'enseignement dispensé associé aux différentes politiques de santé publique menées en France. Les internes ont donc la notion que la fertilité décline tôt mais n'ont probablement pas conscience d'une décroissance aussi rapide. Nous pouvons également constater que même les internes en médecine générale ont des idées reçues, et en particulier, surestiment les capacités de l'AMP en pensant pour pratiquement $90 \%$ d'entre eux - tous sexes confondus - que la fécondation in vitro permet à une femme de concevoir malgré une réserve ovarienne considérablement diminuée. Ce résultat est compatible avec le résultat de plusieurs études où les jeunes femmes et hommes pensent que l'AMP peut surmonter le problème d'une infertilité liée à l'âge [7-9]. Il faut noter par ailleurs qu'environ $1 / 3$ des médecins en formation de notre échantillon conservent de fausses représentations en gynécologie en croyant que la régularité des cycles menstruels est le marqueur d'une réserve ovarienne normale et que les contraceptifs oraux ont des effets délétères sur la réserve ovarienne. De plus, presque $50 \%$ des internes n'ont pas répondu au questionnaire malgré deux relances par e-mails : nous ne connaissons pas leurs caractéristiques mais nous pouvons supposer que le sujet ne les intéressait pas... Les résultats de notre enquête sont probablement le reflet des connaissances des internes déjà les plus sensibilisés au sujet et montrent malgré tout la persistance d'idées reçues au sujet de la fertilité.

\section{Intentions concernant la parentalité}

Les étudiants ont tendance en général à repousser leur projet parental [6], avec dans notre étude, seulement $10,1 \%$ des internes ayant des enfants, alors que plus de 9 internes sur 10 déclaraient en vouloir dans le futur vers 30 ans. Ceci est en accord avec l'étude de Chan et al. [10] où les étudiantes envisageaient d'avoir leur premier enfant aux alentours de 30 ans. Les études de Lampic et al. [11] et de Peterson et al. [11] ont également montré que les étudiants souhaitaient avoir leurs enfants plus tardivement que dans la population générale et que les hommes souhaitaient avoir leur premier enfant plus tard que les femmes. L'implication de l'homme dans le report de la parentalité d'un couple semble donc réelle. Il peut sembler paradoxal que des internes déclarant pour la plupart vouloir avoir des enfants, aient bien conscience de la diminution rapide de la fertilité chez la femme, sans être pour autant prêt à concevoir des enfants plus tôt. Nous nous sommes ainsi aussi intéressés dans notre étude aux internes de sexe masculin, car il semble en pratique clinique que les hommes - chez qui est ancrée l'idée qu'il est possible de " procréer jusqu'à la mort » -, connaissent moins l'urgence procréative que les femmes à partir d'un certain âge. D'ailleurs, l'accès à l'AMP est limité à 43 ans chez les femmes et il n'existe pas de limite légale chez l'homme à l'accès à ces techniques. Il est intéressant de constater dans notre étude que les hommes sont plus favorables que les femmes à l'autoconservation ovocytaire afin de retarder le projet parental du couple.

Il est probable que les internes repoussent leur projet d'enfant pour d'autres raisons que des raisons purement socioéconomiques. Finir ses études est un argument pour plus de 80 \% d'entre eux. Si les raisons financières sont avancées pour la moitié d'entre eux, le désir de garder une mobilité et de développer d'autres d'intérêts est aussi décrit pour environ deux tiers d'entre eux. Il est cependant étonnant qu'une population de médecins n'ait pas un comportement différent de ce que l'on appréhende en population générale, car on aurait pu supposer qu'une bonne connaissance des problèmes de fertilité liés à l'âge féminin, pourrait avoir une incidence sur les choix de vie.

Presque 2/3 des internes interrogés estimaient n'être pas assez informés des moyens mis à leur disposition (stage en surnombre, disponibilités, congés maternités-paternités) pour favoriser un projet de parentalité pendant leur internat. Un programme d'information et de sensibilisation pourrait être mis en place dans les universités en niveau Master. L'étude américaine de Clark et al. [12] a étudié a posteriori la fertilité de 329 femmes médecins âgées en moyenne de 42,7 ans ayant obtenu leur diplôme de médecine entre les années 1995 et 2000 : 25 \% d'entre elles avaient eu un diagnostic d'infertilité dont $36,8 \%$ en rapport avec une baisse de la réserve ovarienne liée à l'âge. Ces femmes ont estimé que 65,9\% des décisions qu'elles avaient prises concernant leur projet parental avaient été influencé par leur carrière. Rétrospectivement, 27,9 \% des femmes auraient tenté de concevoir plus tôt, 17,7 \% auraient choisi une spécialité différente et 7,2\% d'entre elles auraient eu recours à une autoconservation ovocytaire.

\section{Comportement présumé en cas de baisse de la réserve ovarienne}

Dans l'hypothèse d'un diagnostic de baisse de réserve ovarienne, presque trois quarts des internes féminines approuveraient le fait d'avoir un enfant plus tôt mais les avis restent partagés concernant le recours à une autoconservation d'ovocytes puisque moins d'une sur deux y serait favorable. Le comportement présumé des hommes devant l'annonce d'un diagnostic de baisse de la fertilité chez leur compagne a été peu étudié dans la littérature médicale. Ainsi dans notre étude, deux tiers des internes masculins étaient favorables pour avoir des enfants plus tôt que prévu en cas de diminution de la réserve ovarienne de leur compagne et presque un interne sur deux conseilleraient à leur compagne de réaliser une 
autoconservation ovocytaire (groupes tout à fait d'accord et d'accord). Nos résultats sont concordants avec ceux de l'étude d'Azhar et al. [13] qui a montré que dans une situation de réserve ovarienne altérée, 48 \% des professionnels de la santé essaieraient de concevoir un enfant plus tôt que prévu, $21 \%$ choisiraient une autoconservation des ovocytes et $7 \%$ essaieraient de trouver un partenaire plus rapidement. Pour seulement $14 \%$ d'entre eux, une telle annonce n'aurait aucune incidence sur leur projet de vie.

L'autoconservation des ovocytes pour convenance personnelle fait actuellement débat en France alors qu'elle est autorisée dans de nombreux pays [14]. En France, elle est autorisée pour raison médicale en cas de traitement ou de pathologie risquant d'affecter la fertilité. Le Collège national des gynécologues et obstétriciens français (CNGOF) a d'ailleurs émis un avis favorable à l'autorisation de l'autoconservation ovocytaire dite " sociétale » en 2012 [15]. La plupart des pays industrialisés sont confrontés à la problématique du report de conception du premier enfant d'origine sociétale et à l'augmentation du taux d'infertilité liée à l'âge. Certains pays comme les États-Unis et la Grande Bretagne ont déjà entrepris des campagnes publicitaires d'informations afin de sensibiliser les jeunes couples et les professionnels de santé [16]. En France, le CNGOF a demandé, en 2009, par le biais d'un communiqué que tous les professionnels informent les femmes sur la chute de la fertilité avec l'âge. L'agence de la biomédecine souhaite quant à elle que l'on incite les femmes et les hommes à concevoir avant 35 ans (voire mieux avant 30 ans s'ils souhaitent plusieurs enfants) et rappelle que "l'AMP n'est pas une baguette magique lorsque l'âge maternel augmente » [17].

L'étude a été réalisée auprès d'internes de médecine générale d'une seule faculté et il aurait été intéressant d'augmenter l'effectif de la population étudiée en analysant des internes appartenant à d'autres facultés de la région Provence-Alpes-Côte d'Azur (région où se concentrent beaucoup de diplômés du supérieur). Une étude analysant les internes d'autres spécialités médicales ou chirurgicales où le nombre d'années d'études est supérieur à celui des internes de médecine générale serait également intéressante pour apprécier les intentions de report de projet parental. De plus, malgré nos sollicitations, le taux de réponse des hommes a été inférieur à celui des femmes, laissant supposer un biais de recrutement : les hommes qui ont participé à notre étude étaient probablement plus sensibilisés à la problématique de la fertilité. Les femmes ont davantage répondu aux questionnaires car elles sont probablement plus intéressées par la problématique de leur future fertilité, comme cela avait été observé dans l'étude de Bunting et al. [18].

\section{Conclusion}

Malgré quelques idées reçues et tenaces comme par exemple l'effet délétère des pilules contraceptives et la normalité de la réserve ovarienne si il y a des règles spontanées, les internes de médecine générale ayant participé à notre étude disposaient de bonnes connaissances sur la fertilité et la réserve ovarienne. Ils sous-estimaient cependant la décroissance rapide de la fertilité féminine avec l'âge et surestimaient l'efficacité de l'AMP pour y pallier. La plupart des internes envisagent de concevoir leurs enfants après la fin des études médicales, bien que les connaissances sur le déclin de la fertilité de la femme avec l'âge sont globalement acquises. Il serait intéressant d'étudier par une étude qualitative en sciences humaines et sociales, les freins socioéconomiques des internes qui les conduisent à reporter leur projet de parentalité malgré le risque connu d'hypofertilité. Quels sont les facteurs qui font qu'une information perçue n'est pas une information qui permet un changement de pratique? Une campagne d'information et de sensibilisation auprès des étudiants de l'enseignement supérieur serait souhaitable afin qu'ils aient davantage conscience des conséquences de l'âge sur la fertilité et aient la possibilité d'organiser leur projet de vie en conséquence.

\section{Déclaration de liens d'intérêts}

Les auteurs déclarent ne pas avoir de liens d'intérêts.

\section{Remerciements}

Cette étude s'insère dans le programme investissements d'avenir géré par l'Agence nationale de la recherche (projet Creer A*Midex ; n ${ }^{\circ}$ ANR-11-IDEX-0001-02).

\section{Références}

[1] Broekmans FJ, Knauff EAH, te Velde ER, Macklon NS, Fauser BC. Female reproductive ageing: current knowledge and future trends. Trends Endocrinol Metab 2007;18:58-65.

[2] Faddy MJ, Gosden RG. Ovary and ovulation: a model conforming the decline in follicle numbers to the age of menopause in women. Hum Reprod 1996;11:1484-6.

[3] Belaisch-Allart J, Maget V, Mayenga J-M, Grefenstette I, Chouraqui A, Belaid Y, et al. Prendre en charge les femmes de 40 ans et plus en FIV : pour ou contre ou pour et contre ? Gynecol Obstet Fertil 2015;43:599-603

[4] Vialle M, Perrin J, Amar-Hoffet A, Boyer P, Courbiere B. Femmes infertiles de plus de 40 ans : loin du mythe de la femme " carriériste " et du " droit à l'enfant ». Gynecol Obstet Fertil 2016;44:225-31.

[5] Leridon $\mathrm{H}$. Can assisted reproduction technology compensate for the natural decline in fertility with age?. A model assessment. Hum Reprod 2004;19:1548-53.

[6] Insee - Population - Un premier enfant à 28 ans. n.d. http://www.insee.fr/fr/ themes/document.asp?ref id=ip1419.(accessed January 31, 2015).

[7] Nouri K, Huber D, Walch K, Promberger R, Buerkle B, Ott J, et al. Fertility awareness among medical and non-medical students: a case-control study. Reprod Biol Endocrinol RBE 2014;12:94.

[8] Lampic C, Svanberg AS, Karlström P, Tydén T. Fertility awareness, intentions concerning childbearing, and attitudes towards parenthood among female and male academics. Hum Reprod 2006;21:558-64.

[9] Bavan B, Porzig E, Baker VL. An assessment of female university students' attitudes toward screening technologies for ovarian reserve. Fertil Steril 2011:96:1195-9.

[10] Chan CHY, Chan THY, Peterson BD, Lampic C, Tam MYJ. Intentions and attitudes towards parenthood and fertility awareness among Chinese university students in Hong Kong: a comparison with Western samples. Hum Reprod 2015;30:364-72.

[11] Peterson BD, Pirritano M, Tucker L, Lampic C. Fertility awareness and parenting attitudes among American male and female undergraduate university students. Hum Reprod 2012;27:1375-82.

[12] Clark NA, Griffith K, Perkins E, Jagsi R. Experiences with fertility and infertility among generation X physicians. Fertil Steril 2013;100:S317.

[13] Azhar E, Seifer DB, Melzer K, Ahmed A, Weedon J, Minkoff H. Knowledge of ovarian reserve and reproductive choices. J Assist Reprod Genet 2015;1-7.

[14] Belaisch-Allart J. AMP " sociétale " : pourquoi tant de peur ? Gynecol Obstet Fertil 2014;42:557-8

[15] Collège national des gynécologues et obstétriciens français. L'autoconservation sociétale des ovocytes. 2012. Paris: http://www.cngof.asso.fr/D_TELE/ 121212_autoconservation_ovocytaire_com-press.pdf.

[16] American society for reproductive medicine. Female age-related ferility decline. Fertil Steril 2014;101:633-4.

[17] Agence de la biomédecine. Comment informer sur la baisse de fertilité avec l'âge ?; 2009, https://www.agence-biomedecine.fr/IMG/pdf/fiche4-amp.pdf.

[18] Bunting L, Tsibulsky I, Boivin J. Fertility knowledge and beliefs about fertility treatment: findings from the International fertility decision-making study. Hum Reprod 2013;28:385-97. 